\title{
Rescheduling of Cancelled Elective Surgical Procedures Among Older Adults Post-COVID-19
}

\author{
Kaitlin Gonzalez, BScN(HONS), MD(c), Sabrina Trigo, BHSc(HONS), MD(c), Christine Miller, MPH, MD(c), \\ Diana Urajnik, MA, PhD
}

Northern Ontario School of Medicine, Thunder Bay, ON

https://doi.org/10.5770/cgj.24.485

\begin{abstract}
The COVID-19 pandemic has recently put a stop to elective surgical procedures across Canada, inherently compounding already lengthy waitlists that exist within most disciplines of surgery. These long waits for elective procedures within Canadian provinces have not been caused by the COVID-19 pandemic; it is an acute-on-chronic issue that has been exacerbated by the ongoing COVID-19 pandemic. As hospitals begin to reschedule elective surgeries, patients are likely to be prioritized by clinical urgency using both established and newly created surgical triage severity scales. The objective of this commentary is to discuss issues related to the rebooking of elderly surgical patients during the COVID-19 pandemic within the context of northern medicine. Northern and rural hospitals may already face a multitude of barriers related to the rebooking of surgical patients due to a paucity of available surgical resources, as well as difficulties related to accessing care at the local level. While current surgical rebooking tools have been developed in response to the COVID-19 pandemic, they fail to explore certain risks related to the older adult population which may lead to increased mortality and morbidity. Review of the literature indicates that redistribution of surgical resources for older adults in the COVID-19 era will require consideration of clinical medical ethics vs. population health ethics regarding who should be prioritized in re-bookings for elective surgical procedures. This should be done in conjunction with encompassing surgical triage severity scales specifically made for older adults in the time of COVID-19.
\end{abstract}

Key words: coronavirus, COVID-19, surgical rebooking, frailty, older adult

As a result of the severe acute respiratory syndrome coronavirus 2 (SARS-CoV-2) causing the coronavirus disease of 2019 (COVID-19) pandemic, more than 52,700 hospital procedures were cancelled in the province of Ontario, in addition to hundreds of thousands across Canada. ${ }^{(1)}$ Recent epidemiological modelling has demonstrated that upwards of 28 million surgical procedures will be cancelled or postponed globally due to shifting of hospital resources at the peak of the pandemic. ${ }^{(2)}$ This has led to uncertain wait times for elective and urgent surgical procedures not deemed 'life or limb', thereby impacting the care of a variety of patients around the world. $^{(2)}$ Recently, many provincial health authorities within Canada have initiated relaxation of COVID-19 restrictions and implemented staged plans to resume medical treatment, in conjunction with a slow return to pre-pandemic daily life. These long waits for elective procedures within Canadian provinces have not been caused by the COVID-19 pandemic; rather, it is an acute-on-chronic issue that has been exacerbated by the ongoing COVID-19 pandemic. Re-booking elective surgical procedures has yet to be firmly discussed in any jurisdiction, yet its anticipation brings forth the question as to which patient groups should be prioritized once rebooking commences. A particular patient group of importance in these unprecedented times include older adults who have greatly increased mortality and morbidity rates compared to younger counterparts and who, as a result of the pandemic, may suffer from increased loneliness, social isolation or lack of supports. ${ }^{(3)}$

When considering the re-booking process for surgical patients during COVID-19, hospitals may consider revising existing policies to take into account the severity of patient illness, likelihood of COVID-19 transmission, and/or current resource utilization. ${ }^{(4)}$ Risk factors specifically related to the older adult, however, may be overlooked more easily simply due to the vast number of COVID-19 factors which must now be taken into consideration. Furthermore, re-booking older adults for elective procedures may pose significant challenges for Northern Ontario hospitals where surgical resources may already be scarce. Additional barriers may exist in accessing local health care and travel to distant urban centres may be required to access basic surgical services. ${ }^{(5,6)}$ The complexities of older adult surgical re-booking procedures in the COVID-19 era are intricate, and must be weighed both 
ethically and clinically to ensure equity of health-care delivery, as well as cost-effectiveness. Examining this issue through the lens of medical and clinical ethics will provide guidance for surgeons, hospital booking departments, and health authorities as to how best to redistribute surgical time in the face of the immense backlog caused by the COVID-19 pandemic.

\section{Elderly in the North}

The challenges of accessing treatment during a pandemic are particularly acute in northern and rural areas. The interspersion of small communities in northern areas has resulted in service centralization, often necessitating travel for even the most routine procedures, which can be especially challenging for immobile older adults. Even with the issue of transportation removed, access to timely medical and surgical care in the North is complicated by the fact that only $14 \%$ of general practitioners and $3 \%$ of specialists service these regions of the country. ${ }^{(5)}$ This equates to less availability of both operating rooms and intensive care beds in northern and rural areas across the spectrum of care. Additionally, re-booking of surgical appointments may require a surgeon to re-examine a patient, especially if their health has changed during the course of the pandemic. Barriers complicating re-examination include the following: lack of transportation, hearing loss, cognitive impairment, and inabilities to access virtual care. Similar to many northern areas of Canada, Northern Ontario has a relatively small population dispersed over a large land mass-specifically 780,000 people across $803,000 \mathrm{~km}^{2}{ }^{2}{ }^{(7)}$ Consequently, in the case of COVID-19, the North has not seen adequate distribution of federal subsidy funds within health care due to having fewer 'hot zones' than southern urban areas.

It is well-established that the North has less robust surgical resources and fewer long-term care facilities, including community supports for older adults such as home-care services, meals on wheels, and mobile allied health professionals. ${ }^{(6)}$ COVID-19 physical distancing policies may mean caregivers are unable to visit, run errands or assist their loved ones with completion of basic or instrumental activities of daily life. This poses added challenges for older patients who are discharged and transitioning to living back at home, consequently increasing likelihood of readmission for failure to cope and/or potentially avoidable injuries.

Furthermore, the lack of long-term care beds in northern areas has resulted in more alternative-level-of-care admissions (ALC). Older adults awaiting long-term care placement are assigned the term 'ALC patients'. These patients often spend significant amounts of time admitted as hospital inpatients, yet may have less access to allied health-care services than they would as a patient at a long-term care facility. Due to the COVID-19 pandemic, facilities experiencing an outbreak may ban all non-essential personnel, such as physical therapists, occupational therapists, and speech and language pathologists, for an extended period of time until testing reveals no new COVID-19 cases. This poses long-term risks to an individual's physical and emotional health, especially if loved ones are unable to gain access to the facility as an "essential care partner". Additionally, efficient resource utilization must be considered given the high rates of ALC admissions in the North. A shortage of available hospital beds in rural and remote locations during a pandemic can result in a harmful trickledown effect, with new beds being unavailable to patients for acute and/or life threatening illnesses. Short-term costs may also rise dramatically since acute care is exceedingly more costly than grouped long-term care on a daily basis.

\section{Surgical Re-booking of Elderly Patients}

The re-booking of surgery during COVID-19 is not a simplistic process. There are multiple factors which must be examined, such as the necessity of the proposed surgery, resource utilization during a pandemic, and local COVID-19 transmission rates. While multiple surgical severity scales take into account a patient's age when providing a patient's risk stratification score, surgical site infection risk or postoperative morbidity or mortality rates, and other factors such as frailty, cognition, and functional ability, all also need to be considered during the surgical re-booking process.

Literature demonstrates that frail individuals, who more often than not are elderly, have the highest rate of post-operative complications, regardless of age, surgical discipline, or surgical risk. ${ }^{(8)}$ Current estimates of post-operative complication costs are in excess of $\$ 58,000$ per case, but vary depending on the nature and severity of the complication. ${ }^{(9)}$ Older adult patients deemed as frail, or who have poor functional status or cognitive impairment are also more likely to experience post-operative complications than younger counterparts. (10) Consideration of individual frailty status must, therefore, be considered as an essential component of surgical rebooking processes during COVID-19. Additional factors which may place older adults at a disadvantage during the surgical re-booking process include lack of competency, communication challenges, requirement for caregiving services, and an inability to access reliable transportation.

In 2017, the estimated prevalence of frailty in northern Ontario for the 65-and-older age demographic ranged from 7.8 to $20.2 \%{ }^{(11)}$ Frailty tools have long been used in the practice of geriatrics to complete comprehensive geriatric assessments. Additionally, frailty scales have been adopted by certain areas of medicine, such as emergency departments, in order to recognize underlying risks associated with the elderly. The Fried Frailty Index is one example of a validated assessment tool that could be used pre-operatively. It is based on five pre-defined physical frailty criteria and may aid clinicians in identifying and treating frailty in older adults. (12) An assessment of an individual's frailty status can lead to appropriate services being instituted for the patient, and oftentimes can be administered quite quickly. In the surgical setting, implementation of preoperative frailty screening tools for individuals over the age of 60 is an effective method to identify patients at increased risk of complications, as well as patients who may benefit from medical optimization preoperatively. ${ }^{(8)}$ We believe the addition of a validated frailty 
tool to the surgical re-booking process of elderly patients will allow surgeons, re-booking clerks, and hospitals to ethically and fairly re-book essential surgical procedures of elderly patients during COVID-19.

It must be noted, however, that the inclusion of other factors such as functional status and social support into the development of a senior-friendly surgical re-booking tool would mitigate the risk of post-operative complications. This is because the absence of social support positively predicts an increased 30-day post-operative morbidity risk in older adults. ${ }^{(13)}$

Upon creation of a senior-friendly surgical re-booking protocol, health-care teams should also recognize that a procedure considered elective in a young person may be urgent or even emergent in an older adult. This is highlighted by Steinman et al. ${ }^{(14)}$ where they describe a situation in which an elderly demented patient who was awaiting definitive endoscopic treatment for choledocholithiasis consequently has a percutaneous biliary drain put in place. In this situation the patient's dementia may increase the likelihood of inadvertently dislodging the drain, which may necessitate recurrent emergency room visits. In such circumstances, performing the elective procedure would be preferable to attending multiple visits in the emergency department. Thus, the development of an older adult surgical re-booking tool must also account for appropriately triaging the level of surgical urgency, especially when significant delays may adversely impact the physical or emotional health of an individual.

\section{The Ethical Dilemma}

As SARS-CoV-2 spreads, the surge of persons seeking health care, as well as critically ill patients with SARS-CoV-2, decimated health-care systems globally. Canadian jurisdictions responded to this crisis through diversion and preservation of medical resources, which included the postponement of elective surgical procedures across the nation. Canadian medical authorities have issued statements and recommendations on how individual physicians can best ration resources in the face of a 'new-age' pandemic within Canada, as well as approaches to managing moral distress that may be encountered throughout the pandemic. ${ }^{(15)}$

Moral distress may arise in situations where one understands what the appropriate course of action is ethically, but are unable to carry it out due to external constraints. ${ }^{(15)}$ Consider a situation in which a rural physician has four positive COVID-19 cases all requiring intubation, but the small hospital is only equipped with three ventilators. In this circumstance, the physician may experience moral distress, since the rationing of resources does not allow for the distribution of medical resources according to traditional clinical medical ethics which normally informs health-care system delivery outside the context of a pandemic. In normal circumstances the highest duty of physicians is their commitment to the well-being of their patients. ${ }^{(16)}$ However, pandemic medicine is ruled by population health ethics and utilitarianism, thereby overriding patient-specific duties prescribed by traditional clinical medical ethics. ${ }^{(17)}$
It must be recognized that practicing in a utilitarian manner within remote areas could cause significantly more moral distress among physicians as a result of the community cohesiveness rural physicians serve. For example, as a rural physician there is a high likelihood that you may be faced with decisions about treatment for community members whom you have known your entire life or whom you intereact with on a daily basis. This may be a rare occurrence in large urban centres, but it is a true reality for the rural doctor. In addition, rural physicians are also faced with the knowledge that if they succumb to SARS-CoV-2, there will be potentially no one else to care for the community. ${ }^{(18)}$

\section{Concluding Comments}

The aforementioned sections culminate into one final question which is: Given the unprecedented wait lists for surgical procedures in northern Ontario, should we in this stage of the pandemic shift back to clinical medical ethics and prioritize those with highest mortality and morbidity in order to save these patients' quality years of life, as well as health-care dollars? Or alternatively: Do we adopt population health utilitarianism principles and prioritize younger individuals where there is the potential to save the highest life-years? The answer is still largely unclear, as several jurisdictions continue to raise questions about whether we have already been successful at flattening the curve, or if a second peak will occur. Each institution will need to adopt an individualized approach, ensuring that they consider guidance from government, national and local health authorities, local viral transmission patterns, and discharge barriers and social supports which exist within their community. We do recommend, however, that appropriate surgical re-booking of older adults for elective procedures entail case-by-case assessment with encompassing tools, examining the patient's acuity, social supports post-surgery, their geographic location to the surgical centre, and other impacting factors. Future endeavours beyond this commentary call to the development of truly comprehensive clinical re-booking tools for older adults in the context of the COVID-19 surgical cancellations.

\section{ACKNOWLEDGEMENTS}

This commentary was part of an advocacy project and academic requirement in the NOSM MD Program, supervised by Dr. Diana Uranijuk. Thank you to the Northern Ontario School of Medicine for providing the funding and support of this publication.

\section{CONFLICT OF INTEREST DISCLOSURES}

The authors declare that no conflicts of interest exist.

\section{REFERENCES}

1. Financial Accountability Office of Ontario. Ontario health sector: a preliminary review of the impact of the COVID-19 
outbreak on hospital capacity. Toronto, ON: Financial Accountablility Office; 2020. Retrieved from https://www.fao-on.org/ en/Blog/Publications/health-2020

2. Nepogiev D, Covid Surg Collaborative, Hoste E. Elective surgery cancellations due to the COVID-19 pandemic: global predictive modelling to inform surgical recovery plans. $\mathrm{Br} J$ Surg. 2020; 107(11):1440-1449.

3. Rout N. Risks to the elderly during the coronavirus (COVID-19) pandemic 2019-2020. J Geriatr Care Res. 2020;7(1):27-28.

4. Prachand V, Milner R, Angelos P, et al. Medically necessary, time-sensitive procedures: scoring system to ethically and efficiently manage resource scarcity and provider risk during the COVID-19 pandemic. J Am Coll Surg. 2020;231(2):281-288.

5. Bosco C, Oandasan I. Review of family medicine within rural and remote Canada: Education, practice, and policy. Mississauga, ON: College of Family Physicians of Canada; 2016. Retrieved from https://portal.cfpc.ca/resourcesdocs/ uploadedFiles/Publications/News_Releases/News_Items/ ARFM_BackgroundPaper_Eng_WEB_FINAL.pdf

6. Health Quality Ontario. Health in the North: a report of geography and the health of people in Ontario's two northern regions. Toronto, ON: Health Quality Ontario; 2019. Retrieved from http://healthinthenorth.hqontario.ca/

7. Statistics Canada. Census Profile, 2016 Census. Northern [economic region], Saskatchewan \& Ontario (province). Ottawa, ON: Statistics Canada; 2016. Retrieved from: https:// www12.statcan.gc.ca/census-recensement/2016/dp-pd/prof/ details/page.cfm?Lang $=$ E $\&$ Geo $1=$ ER $\&$ Code $1=4760 \&$ Geo $2=$ PR\&Code $2=35 \&$ Data $=$ Count $\&$ SearchText $=$ North $\&$ SearchType $=$ Begins $\&$ SearchPR $=01 \& \mathrm{~B} 1=$ All

8. Birkelbach O, Mörgeli R, Spies C, et al. Routine frailty assessment predicts postoperative complications in elderly patients across surgical disciplines - a retrospective observational study. BMC Anesthesiol. 2019;19, article number 204. Retrieved from: https://bmcanesthesiol.biomedcentral.com/articles/10.1186/ s12871-019-0880-x

9. Krupka D, Sandberg W, Weeks B. The impact on hospitals of reducing surgical complications suggests many will need shared savings programs with payers. Health Affairs. 2012;31(11).

10. Han B, Qiuping L, Chen X. Effects of the frailty phenotype on post-operative complications in older surgical patients: a sys- tematic review and meta-analysis. BMC Geriatrics. 2019; 19, article number 141. Retrieved from https://link.springer.com/ article/10.1186/s12877-019-1153-8

11. Kehler S, Ferguson T, Stammers AN, et al. Prevalence of frailty in Canadians 18-79 years old in the Canadian Health Measures Survey. BMC Geriatrics. 2017;17:28. Retrieved from: https:// www.ncbi.nlm.nih.gov/pmc/articles/PMC5251297/

12. Op Het Veld L, van Rossum E, Kempen G, et al. Fried phenotype of frailty: cross-sectional comparison of three frailty stages on various health domains. BMC Geriatrics. 2015; 15, article number 77 . Retrieved from: https://bmcgeriatr.biomedcentral .com/articles/10.1186/s12877-015-0078-0

13. Kumar C, Salzman B, Colburn J. Preoperative assessment in older adults: a comprehensive approach. Am Fam Phys. 2018;98(4):214-220. Retrieved from: https://www.aafp.org/ afp/2018/0815/p214.html

14. Steinman M, Perry L, Perissinotto C. Meeting the care needs of older adults isolated at home during the COVID-19 Pandemic [Viewpoint]. JAMA Intern Med. 2020;180(6):819-820.

15. Canadian Medical Association. COVID-19 and moral distress. Ottawa, ON: CMA; 2020. Retrieved from https:// www.cma.ca/sites/default/files/pdf/Moral-Distress-E.pdf? $\mathrm{ga}=2.18033214 .608161944 .1592347546-1145297900.159234754 \overline{6}$

16. Canadian Medical Association. CMA Code of ethics and professionalism. Ottawa, ON: CMA; 2018. Retrieved from: https:// policybase.cma.ca/documents/policypdf/PD19-03.pdf

17. Emanuel E, Persad G, Upshur R, et al. Fair allocation of scarce medical resources in the time of COVID-19. NEngl JMed. 2020; 382:2049-2055. Retrieved from: https://www.nejm.org/doi/full/ 10.1056/NEJMsb2005114

18. Ludwig K, Machnitzke C, Kühlein T. Barriers to practicing General Practice in rural areas-Results of a qualitative prepost-survey about medical students during their final clinical year. GMS J Med Educ. 2018;35(4). Retrieved from: https:// www.ncbi.nlm.nih.gov/pmc/articles/PMC6278231/

Correspondence to: Sabrina Trigo, BHSc(HONS), MD(c), Northern Ontario School of Medicine, 955 Oliver Rd., Thunder Bay, ON, P7B 5E1

E-mail: strigo@nosm.ca 